\section{CRISPR SCREEN IDENTIFIES LOSS OF IFN $\gamma$ R SIGNALING AND DOWNSTREAM ADHESION AS A RESISTANCE MECHANISM TO CAR T-CELL CYTOTOXICITY IN SOLID BUT NOT LIQUID TUMORS}

${ }^{1}$ Rebecca Larson*, ${ }^{2}$ Michael Kann, ${ }^{2}$ Stefanie Bailey, ${ }^{3}$ Nicholas Haradhvala, ${ }^{3}$ Kai Stewart, ${ }^{2}$ Amanda Bouffard, ${ }^{2}$ Irene Scarfo, ${ }^{2}$ Mark Leick, ${ }^{2}$ Trisha Berger, ${ }^{2}$ Max Jan, ${ }^{3}$ Julia Joung, ${ }^{3}$ Tamara Ouspenskaia, ${ }^{3}$ Travis Law, ${ }^{3}$ Aviv Regev, ${ }^{1}$ Gad Getz, ${ }^{1}$ Marcela Maus. ${ }^{1}$ Harvard University, Cambridge, MA, USA; Massachusetts General Hospital, Boston, MA, USA; ${ }^{2}$ Massachusettes General Hospital, Boston, MA, USA; ${ }^{3}$ Broad Institute of MIT and Harvard, Cambridge, MA, USA

Background Chimeric Antigen Receptor (CAR) therapy has had a transformative impact on the treatment of hematologic malignancies ${ }^{1-6}$ but success in solid tumors remains elusive. We hypothesized solid tumors have cell-intrinsic resistance mechanisms to CAR T-cell cytotoxicity.

Methods To systematically identify resistance pathways, we conducted a genome-wide CRISPR knockout screen in glioblastoma cells, a disease where CAR T-cells have had limited efficacy. ${ }^{7}{ }^{8}$ We utilized the glioblastoma cell line U87 and targeted endogenously expressed EGFR with CAR T-cells generated from 6 normal donors for the screen. We validated findings in vitro and in vivo across a variety of human tumors and CAR T-cell antigens.

Results Loss of genes in the interferon gamma receptor (IFN $\gamma \mathrm{R}$ ) signaling pathway (IFN $\gamma \mathrm{R} 1, \mathrm{JAK} 1, \mathrm{JAK} 2)$ rendered U87 cells resistant to CAR T-cell killing in vitro. IFN $\gamma \mathrm{R} 1$ knockout tumors also showed resistance to CAR $\mathrm{T}$ cell treatment in vivo in a second glioblastoma line U251 in an orthotopic model. This phenomenon was irrespective of CAR target as we also observed resistance with IL13Ralpha2 CAR T-cells. In addition, resistance to CAR T-cell cytotoxicity through loss of IFN $\gamma \mathrm{R} 1$ applied more broadly to solid tumors as pancreatic cell lines targeted with either Mesothelin or EGFR CAR Tcells also showed resistance. However, loss of IFN $\gamma \mathrm{R}$ signaling did not impact sensitivity of liquid tumor lines (leukemia, lymphoma or multiple myeloma) to CAR T-cells in vitro or in an orthotopic model of leukemia treated with CD19 CAR. We isolated the effects of decreased cytotoxicity of IFN $\gamma \mathrm{R} 1$ knockout glioblastoma tumors to be cancer-cell intrinsic because CAR T-cells had no observable differences in proliferation, activation (CD69 and LFA-1), or degranulation (CD107a) when exposed to wildtype versus knockout tumors. Using transcriptional profiling, we determined that glioblastoma cells lacking IFN $\gamma \mathrm{R} 1$ had lower upregulation of cell adhesion pathways compared to wildtype glioblastoma cells after exposure to CAR T-cells. We found that loss of IFN $\gamma \mathrm{R} 1$ reduced CAR T-cell binding avidity to glioblastoma.

Conclusions The critical role of IFN $\gamma \mathrm{R}$ signaling for susceptibility of solid tumors to CAR T-cells is surprising given that CAR T-cells do not require traditional antigen-presentation pathways. Instead, in glioblastoma tumors, IFN $\gamma \mathrm{R}$ signaling was required for sufficient adhesion of CAR T-cells to mediate productive cytotoxicity. Our work demonstrates that liquid and solid tumors differ in their interactions with CAR T-cells and suggests that enhancing T-cell/tumor interactions may yield improved responses in solid tumors.

Acknowledgements RCL was supported by T32 GM007306, T32 AI007529, and the Richard N. Cross Fund. ML was supported by T32 2T32CA071345-21A1. SRB was supported by T32CA009216-38. NJH was supported by the Landry Cancer Biology Fellowship. JJ is supported by a NIH F31 fellowship (1F31-MH117886). GG was partially funded by the Paul C. Zamecnik Chair in Oncology at the Massachusetts General
Hospital Cancer Center and NIH R01CA 252940. MVM and this work is supported by the Damon Runyon Cancer Research Foundation, Stand Up to Cancer, NIH R01CA 252940, R01CA238268, and R01CA249062.

\section{REFERENCES}

1.. Maude SL, et al. Tisagenlecleucel in children and young adults with B-cell lymphoblastic leukemia. N Engl J Med 2018;378:439-448

2.. Neelapu

SS, et al. Axicabtagene ciloleucel CAR T-cell therapy in refractory large B-cell lymphoma. N Engl I Med 2017;377:2531-2544.

3.. Locke $\mathrm{FL}$, et al. Long-term safety and activity of axicabtagene ciloleucel in refractory large B-cell lymphoma (ZUMA-1): a single-arm, multicentre, phase 1-2 trial. The Lancet Oncology 2019;20:31-42.

4.. Schuster $\mathrm{SJ}$, et al. Chimeric antigen receptor T cells in refractory B-cell lymphomas. N Engl J Med 2017:377:2545-2554.

5.. Wang $M$, et al. KTE-X19 CAR T-cell therapy in relapsed or refractory mantle-cell lymphoma. N Engl J Med 2020;382:1331-1342.

6. Cohen $A D$, et al. B cell maturation antigen-specific CAR T cells are clinically active in multiple myeloma. J Clin Invest 2019;129:2210-2221.

7.. Bagley SJ, et al. CAR T-cell therapy for glioblastoma: recent clinical advances and future challenges. Neuro-oncology 2018;20:1429-1438.

8.. Choi $\mathrm{BD}$, et al. Engineering chimeric antigen receptor $\mathrm{T}$ cells to treat glioblastoma. J Target Ther Cancer 2017;6:22-25.

Ethics Approval All human samples were obtained with informed consent and following institutional guidelines under protocols approved by the Institutional Review Boards (IRBs) at the Massachusetts General Hospital (2016P001219). Animal work was performed according to protocols approved by the Institutional Animal Care and Use Committee (IACUC) (2015N000218 and 2020N000114).

http://dx.doi.org/10.1136/jitc-2021-SITC2021.221 